\title{
Bridging the Knowledge Gap in Transmission-Blocking Immunity to Malaria: Deciphering Molecular Mechanisms in Mosquitoes
}

\author{
Sandeep Satapathy', Andrew W. Taylor-Robinson ${ }^{2 *}$ \\ ${ }^{1}$ Department of Biological Sciences, Indian Institute of Science Education and Research, \\ Bhopal, India \\ ${ }^{2}$ School of Medical \& Applied Sciences, Central Queensland University, Rockhampton, Australia \\ Email: *a.taylor-robinson@cqu.edu.au
}

Received 15 March 2016; accepted 23 May 2016; published 26 May 2016

Copyright (C) 2016 by authors and Scientific Research Publishing Inc.

This work is licensed under the Creative Commons Attribution International License (CC BY). http://creativecommons.org/licenses/by/4.0/

(c) (i) Open Access

\begin{abstract}
The worldwide decline over the last decade in the number of clinical cases of malaria does not mean an end to the universal problem of malaria pathogenesis in those afflicted by infection. Resistance to drugs, higher risk of disease relapse and failure to maintain effective memory of the pathogen in the absence of persistent exposure result in the repeated failure of anti-malarial treatments. The artificial blocking of transmission of the Plasmodium parasite between hosts from human to Anopheles mosquito, and vice versa, is crucial to restricting the spread of disease. However, a limited knowledge of the molecular mechanisms in operation for transmission of malaria has impeded progress towards a transmission-blocking vaccine. This review highlights the role of anti-malarial immune responses to antigen-specific targets for designing effective vaccines against the sexual stages of Plasmodium that occur within the invertebrate vector. In particular, artificial induction of gametocyte and ookinete apoptosis as a novel means to prevent gamete fertilization and oocyte development, respectively, is highlighted. This and other recent insights into our understanding of the molecular regulation of transmission-blocking immunity are discussed and future prospects considered.
\end{abstract}

\section{Keywords}

Malaria, Parasite, Sexual Stage, Transmission-Blocking, Vaccine, Apoptosis

\footnotetext{
${ }^{*}$ Corresponding author.
}

How to cite this paper: Satapathy, S. and Taylor-Robinson, A.W. (2016) Bridging the Knowledge Gap in Transmission-Blocking Immunity to Malaria: Deciphering Molecular Mechanisms in Mosquitoes. Advances in Infectious Diseases, 6, 33-41. http://dx.doi.org/10.4236/aid.2016.62005 


\section{Introduction}

Malaria thrives in diverse climatic conditions ranging from temperate to tropical regions, such that this important parasitic disease of humans is currently reported to occur in over one hundred countries around the world. Of the estimated global population of 7.3 billion people [1], 3.4 - 3.7 billion are at risk of contracting malaria [2] [3]. It is routinely claimed that there are 300 - 500 million clinical cases worldwide annually [4]. The 1 - 3 million deaths that result equate to a death every 10 - 30 seconds [5]. In terms of infection control, arguably as alarming is the statistical measure of the human population infected with malaria parasites, estimated to be around 1.5 billion [2]. The distribution of malaria is mainly among socioeconomically lower developed countries, further perpetuating their cycle of poverty. It is calculated to cost these endemic nations approximately US $\$ 12$ billion a year [3]. Annual losses of 1.3\% in global economic growth may be attributed in part to lost person hours and therefore to reduced productivity [6]. Furthermore, children who miss school suffer from an education deficit which ultimately affects their future employment prospects. Against this depressing backdrop, the World Health Organization has provided a measure of hope by revealing a global decline in the number of malaria cases in the past 15 years [2]. However, since the parasite has evaded successfully multiple attempts at control and eradication over several decades still novel approaches are required in order to elucidate fully the "what, why and how" of the malaria life cycle, transmission and infection [7]. Only then will researchers be best equipped to combat this major public health scourge.

\section{Malaria: Parasite and Infection}

Malaria is a vector-borne disease transmitted by female Anopheles mosquitoes and which causes an acute or relapsing infection of protozoan parasites of the genus Plasmodium [8]. P. falciparum, P. vivax, P. malariae, $P$. ovale and $P$. knowlesi are the five species identified to produce clinical outcomes in humans. Of these, $P$. falciparum and $P$. vivax cause the most mortality and morbidity, respectively. Heterogeneity among species leads to different clinical symptoms [8]. Lethality is associated with increased risk of parasite resistance developing to current anti-malarial drugs [9].

Species, strain and antigen specificity of the host immune response, compounded by clonal antigenic variation and geographical antigenic diversity, presents considerable challenges to the design of an effective malaria vaccine for prophylactic or therapeutic use in humans [7]. Nevertheless, candidate vaccines as well as available chemotherapy have met with qualified success in combating genetically related strains of Plasmodium. A compounding factor is that mixed infections of $P$. falciparum and $P$. vivax can take place within the one host [10]. However, it is not known which dominates and how the mode of infection varies in different individuals [11]. Since these distinct parasites share a common vector, from an evolutionary perspective such co-operative propagation of species suggests a mutual benefit from within-host competition [12].

\section{Malaria: Life Cycle and Stage-Specific Immunity}

Plasmodium progresses through multiple transitions in alternating hosts, sexual reproduction taking place in the midgut of an Anopheles mosquito and asexual replication occur in the liver and peripheral blood of a vertebrate, including humans (Figure 1). Infection within the liver is immunologically and clinically quiescent, disease pathogenesis occurring only when schizonts are released from the liver into the circulatory blood. It is the subsequent repeated cycle of intraerythrocytic vegetative growth and host cell rupture that gives rises to the characteristic influenza-like, paroxysmal symptoms of uncomplicated infection [8].

When a mosquito ingests a blood meal from a malaria-infected person, male and female gametocyte stage parasites enter the midgut where they are enclosed by a newly synthesized peritrophic matrix. Intracellular male gametocytes exit their erythrocytic environment through a process of exflagellation that is triggered cumulatively by mosquito-derived xanthurenic acid, a lowering of body temperature and a commensurate rise in $\mathrm{pH}$, whereupon fertilization soon follows. Within 24 hours a diploid zygote forms and further development and differentiation results in banana-shaped, motile ookinetes. From the midgut ookinetes penetrate the epithelium, reach the basal lamina and differentiate into oocysts. Multiplication through mitotic divisions over 7 - 15 days may result in tens of thousands of motile, haploid sporozoites [13]. These migrate to the salivary glands and are injected into an individual's body when the now infectious mosquito next bites a human. This releases several sporozoites into the skin, where they may reside for up to six hours before migrating to other cellular locations 


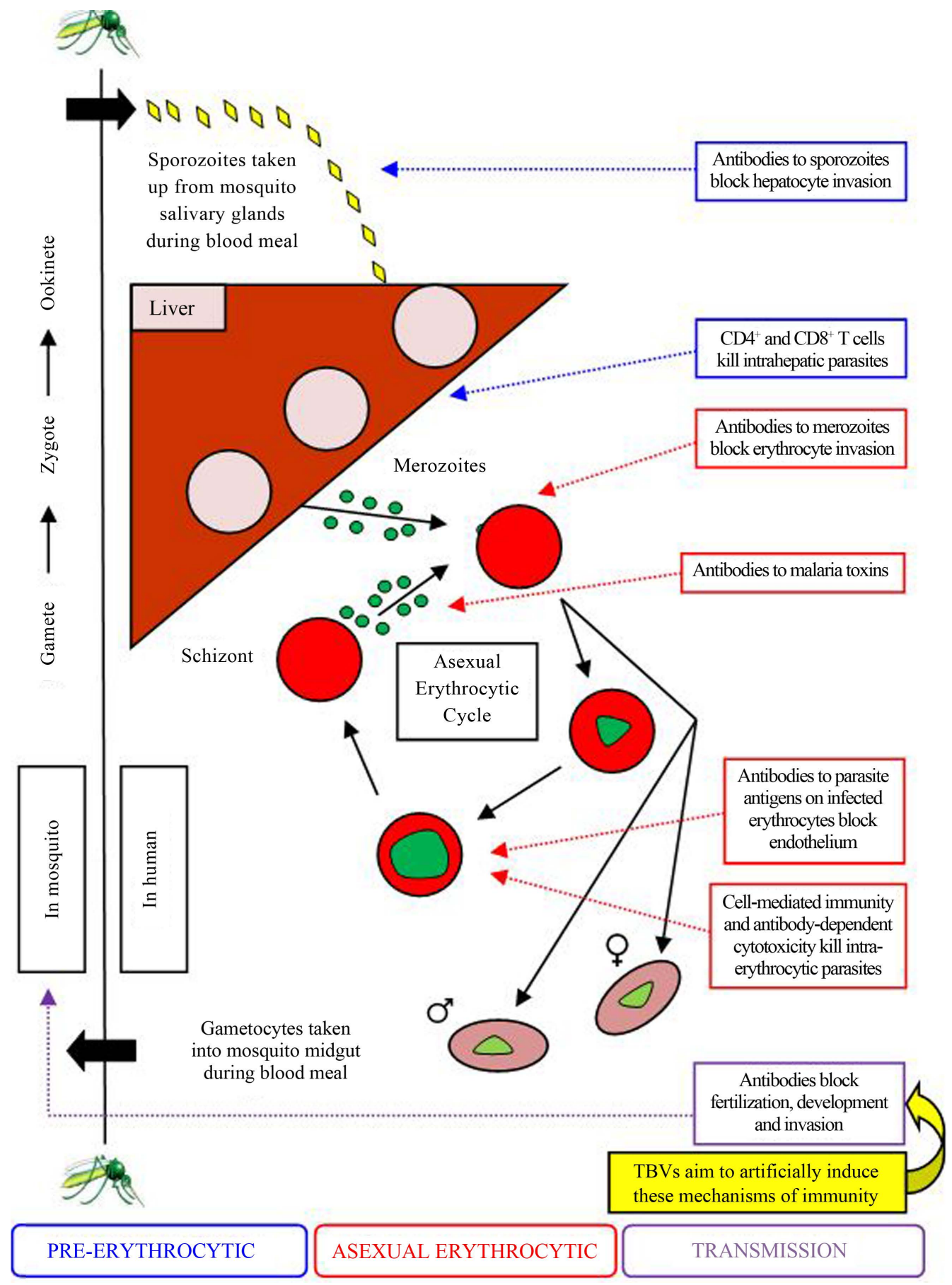

Figure 1. Schematic outline of stages of infection of the major human malaria parasite Plasmodium falciparum, highlighting antibody-mediated mechanisms of immunity against the sexual stages that transmission-blocking vaccines aim to induce.

[14]. The parasite load released per mosquito bite, on average around a hundred sporozoites, determines the level of infectivity [15].

Typically, around one third of sporozoites leave the skin and enter the lymphatic system while the remainder migrate to hepatocytes via the bloodstream [16]. In the liver, sporozoites reach specialized reticuloendothelial cells, Kupffer cells, where they undergo rapid asexual amplification to produce exoerythrocytic schizonts. This process of schizogony takes between 2 - 10 days, depending on the species of Plasmodium [17]. Many mero- 
zoites form which are released back into the blood, invade erythrocytes and undergo a cycle of asexual replication that repeats every 46 - 48 hours for $P$. falciparum, the deadliest of the human malaria species. A minority of merozoites leave this cycle and undergo sexual development into male and female gametocytes that are ready to transfer to a mosquito when it feeds on that person [13].

At each stage different antigen-specific immunological responses perform protective (and possibly immunopathological), sterilizing and altruistic effects (Figure 1). Harnessing each form of specific immunity plays a role in the overall strategy to control malaria, such that potentiation of a protective response underpins rational vaccine design [18]-[20]. The primary focus of this review is to discuss how by manipulating the molecular machinery of Plasmodium sexual differentiation transmission of gametes from a vertebrate host to the mosquito may be blocked, thereby providing a premise for transmission-blocking vaccines.

\section{Regulation of Immunity and Potential for Blocking Transmission}

Persons exposed persistently to malaria infections possess elevated levels of pro-inflammatory cytokines and complement components in their peripheral blood, which are ingested by a mosquito when feeding on an infected host [21]. This process leads to uptake both of sexual stage of parasites (gametocytes) and reactive immune molecules that negate the propagation and further differentiation of those gametocytes [21] [22] (Figure 2). This mechanism may achieve an effective blockade of transmission to the mosquito by activation of the immune molecules co-ingested in the blood meal [23].

Among different pro-inflammatory cytokines that are stimulated by malaria infection, tumour necrosis factor-alpha (TNF- $\alpha$ ) and interferon gamma (IFN- $\gamma$ ) rise appreciably with increased parasitic burden [23] (Figure 2). Secreted by peripheral blood mononuclear cells, these cytokines cause a decline in gametocytic infectivity as the sexual stage parasites cannot infect the mosquito effectively due to their activity. An elevated concentration of TNF- $\alpha$ is also associated with upregulation of extracellular signal-mediated apoptosis and thus may provide a promising field of research for parasite transmission blockade (Figure 2).

\section{Molecular Biology of Gametogenesis}

Gametocytes develop within 2 - 10 days of primary malaria infection of a vertebrate host. Broadly, gametocyte development can be classified into five stages depending on the shape of the gametes and their genomics and proteomics [24] [25]. Each gametocyte resides within a host erythrocyte which it forces to adopt a crescent shape to accommodate it. The gametocyte is protected from the surrounding erythrocyte by a parasitophorous vacuolar membrane located next to the parasitic plasma membrane [26].
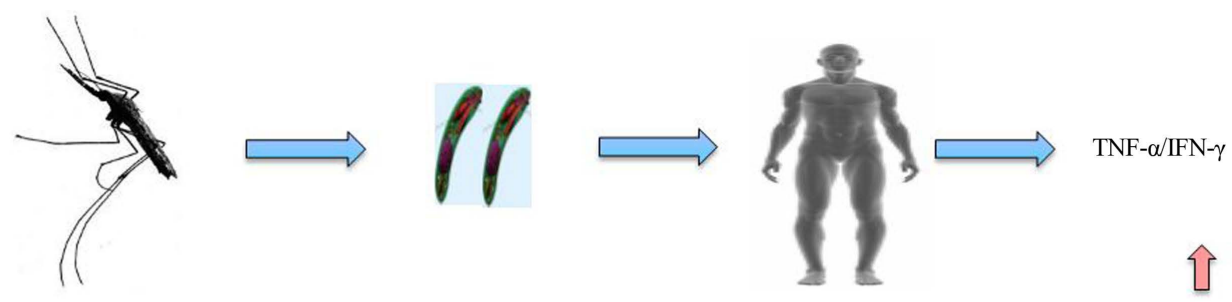

Lower parasite exposure Insufficient sporozoites released to host No clinical manifestation of malaria Usual expression of cytokines

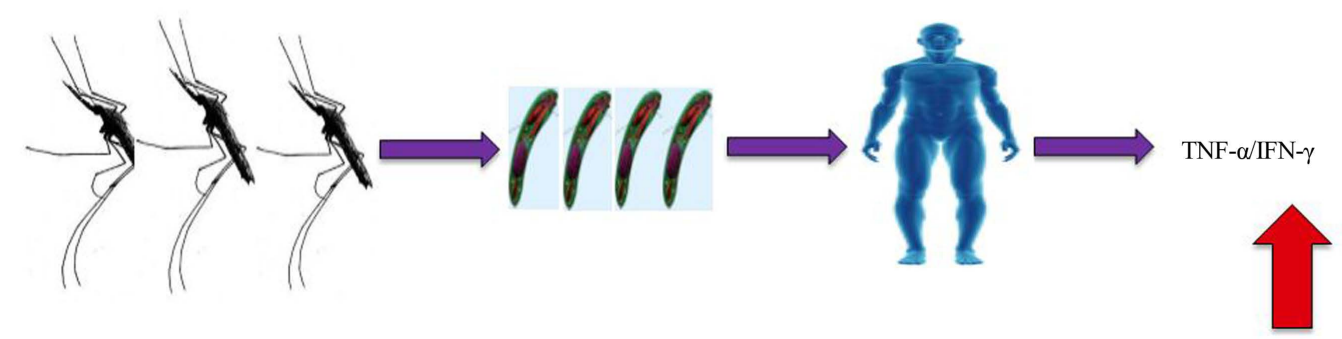

Higher parasite exposure Sufficient sporozoites released to host Clinical manifestation of malaria Elevated expression of cytokines

Figure 2. Schematic representation of upregulation of pro-inflammatory cytokines in response to clinically manifested malaria. 
Gametocyte development in the vertebrate host is restricted at the G0 phase of the cell cycle and further cell differentiation takes place only when it enters the mosquito midgut [22]. Once there, a lowering of temperature by around $5^{\circ} \mathrm{C}$, the presence of the mosquito-derived molecule xanthurenic acid and a rise of $\mathrm{pH}$ to 8 combine toactivate gametocytes [27]. Gametocyte development is measured by exflagellation of male gametes around 15 minutes after exposure to the above mosquito-mediated activation factors [24]. During activation of gamete formation there is a sudden rise in levels of intracellular calcium and cyclic guanosine monophosphates [28]. Propagation of sexual stage parasites involves upregulated expression of two integral membrane proteins, guanylyl cyclases $-\alpha$ and $-\beta$ [29].

\section{Current Transmission-Blocking Vaccines (TBVs)}

Most current anti-malarial strategies aim to reduce clinical manifestations of the disease by lowering parasitaemia and decreasing parasitic density [30]. An increased parasite burden is facilitated by an immune compromised host and/or evasion of host immunity. Thus, determining deficits in the anti-malarial immune response may shed light on the parasite's strategies for propagation within the host.

An understanding of naturally acquired immunity and the role of premunition in eliciting a host response to Plasmodium has enabled considerable progress in designing vaccines against malaria by targeting the surface molecules of the parasite [31]. The major focus has been:

1) Control of disease via anti-malarial immunity, with lower morbidity and mortality rates;

2) Control of parasite propagation and parasite density leading to decreased pathogen burden.

In order to achieve both these goals to control sexual stage transmission of the parasite from an infected host to a healthy host, there is a need to identify novel antigenic targets represented by parasite surface proteins, especially those expressed by the gamete stage or during ookinete to oocyte differentiation [30]. However, a major consideration is the species specificity of the vaccine-induced immune reactivity although this should aim to be equally effective against different genetically related strains or isolates within a species.

The finding that serum antibodies target surface antigens of extracellular gametocytes has been helpful in achieving blockade of parasite transmission in vertebrate hosts via malaria-infected mosquitoes [32] (Figure 3).

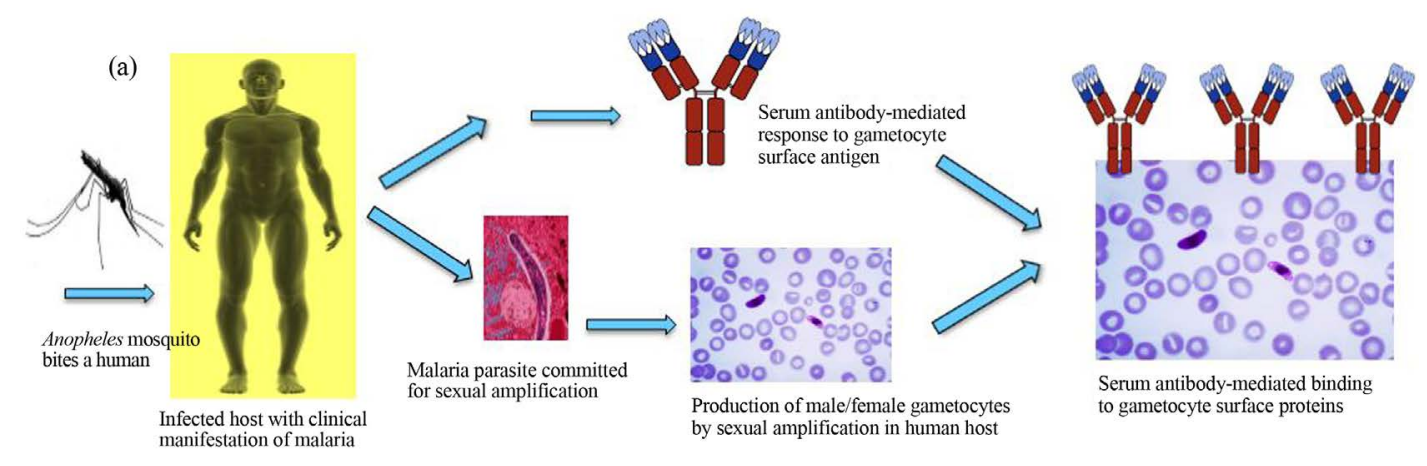

(b)
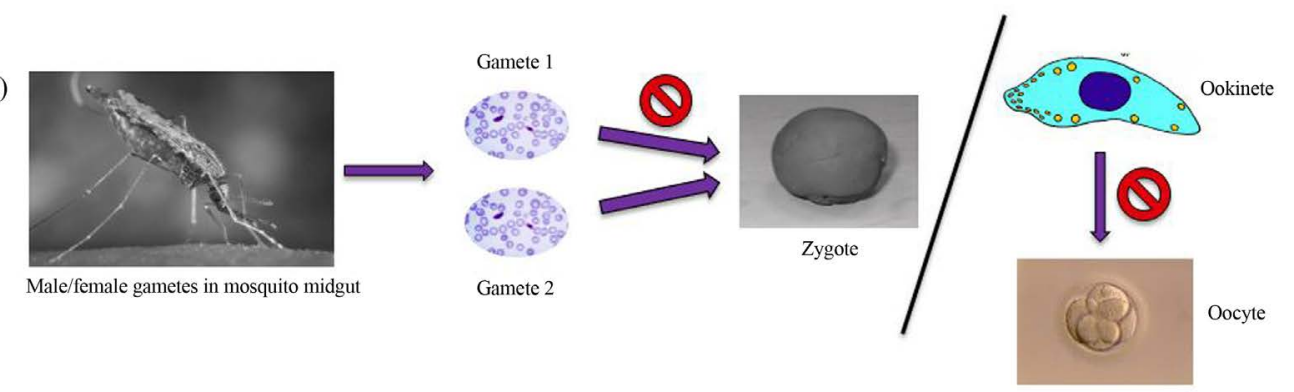

Figure 3. Rationale for transmission-blockage vaccine design. (a) Serum antibody-mediated response targets gametocyte surface antigens. (b) Blockade of parasite fertilization in the midgut, with consequent prevention of differentiation of ookinete into oocyte. 
These antibodies are effective in eliciting a protective immune response during primary parasitic infection. Even during subsequent infection serum-mediated blockade of parasite transmission to the mosquito is pronounced [32] (Figure 3). This has led to the emergence of a separate focus for vaccine development against malaria, that of TBVs [33] [34].

Apart from targeting parasite surface antigens, much research effort is being channeled into trying to elucidate mechanisms to block parasite fertilization in the midgut. If successful, this would prevent formation of the zygote that matures to give rise to infective sporozoites, thereby providing a novel rationale for design of anti-malarial vaccines. One focus of this strategy is to induce blockade of the apoptosis of ookinetes, the mechanism involved in differentiation and development of oocytes, the parasite stage which invades the outer gut surface of the mosquito [35].

\section{Apoptosis of Malaria Sexual Stages}

Apoptosis or programmed cell death involves induction of self-destruction of cells, leading to clearance of infection-associated cellular debris and also at times to de novo development of cells and tissues [36] [37]. The role of apoptosis in parasite propagation is poorly defined. Isolated slivers of information on apoptosis suggest a possible role for apoptosis in blocking transmission to the malaria mosquito from an infected vertebrate host [38] [39].

Research into apoptosis has examined Plasmodium gametocyte development and sex determination [40]. During differentiation into male and female gametocytes genetic clones commit to more male gametocytes and thereby apoptose female gametocytes [41] [42]. This characteristic feature of gametocyte development is known to increase the infectivity of each parasite clone [43]. However, such a model of shared evolution among malaria parasites indicates a competitive selection pressure which drives the advantage of a higher chance of infection [44] [45].

In addition to the putative part played by apoptosis in sex determination of gametocytes, apoptosis is reported to occur during development of oocytes from ookinetes in the mosquito midgut [46] [47]. However, it is interesting to note that this form of apoptosis promotes rather than restricts parasite propagation. Simultaneously, in the host peripheral blood there is an upregulation of TNF- $\alpha$, which is ingested by the mosquito along with gametes. Thus, as TNF- $\alpha$ is known to induce extracellular ligand-mediated apoptosis it is important to understand the role of this pro-inflammatory cytokine in regulating apoptosis in the mosquito midgut [48].

\subsection{Hypothesis for Arresting Sexual Stage Propagation}

1) Over expression of TNF- $\alpha / \mathrm{IFN}-\gamma$ in the host peripheral blood during parasitic infection interferes with gametocyte cell cycle arrest and thus can block transmission of sexual stages of Plasmodium to the Anopheles mosquito.

2) Upregulated TNF- $\alpha$ in the bloodstream of the vertebrate host limits gametocyte infectivity by inducing cell apoptosis (arrested in the G0 phase).

3) Inhibition of apoptosis (i.e. inhibition of initiator and executioner caspases [49]) can block the development of ookinetes to oocytes in the mosquito midgut.

4) Although TNF- $\alpha$ is over expressed in the host prior to vertebrate transmission TNF- $\alpha$-mediated apoptosis is inhibited. This may be due to the possible inhibition of apoptosome formation (facilitated by cytochrome c and apoptotic protease-activating factor 1 ).

\subsection{Relevance of Model to Blocking Transmission}

In order to realign a consolidated course of action that aims to effectively combat malaria, three principal outcomes of prior programs can be summarized:

1) Development of resistance by parasites to anti-malarial drugs and a higher risk of disease relapse, especially for $P$. falciparum and $P$. vivax;

2) A major breakdown of vector control programs, especially in tropical and subtropical countries;

3) Limitation to vaccine development targeting Plasmodium molecular moieties on the surface of mature erythrocyte stage parasites, gametes and sporozoites.

Overcoming the challenge of each of these roadblocks to controlling malaria is predicated on the realization that a lack of insight into the molecular mechanism(s) for arresting asexual and sexual propagation of parasites 
has worsened the scenario. Although available chemotherapeutic regimens limit asexual stage parasitaemia, notwithstanding growing drug resistance, sexual stage propagation in invertebrate hosts still remains largely unexplored and therefore of untapped potential as a target for intervention [20].

\section{Future Perspectives}

Bridging the gap in knowledge relating to apoptosis of Plasmodium will underpin attempts to block transmission of malaria to the Anopheles mosquito. The successful prevention of differentiation of ookinetes into oocytes by apoptosis will inhibit propagation of sexual stages of the malaria parasite, thereby helping to reduce or even eliminate parasite transmission from an infected host to a healthy host via an intermediary Anopheles mosquito.

Advancing from our present understanding of how parasite burden can be lowered, targeting the molecular machinery of parasite propagation (both for sexual and asexual stages) may provide a novel strategy for malaria vaccine development [50]. In this context, a cohort of vertebrate host immune responses that includes upregulation of pro-inflammatory cytokines, mast cell degranulation, vasodilation of hepatocytes enabling higher infiltration of neutrophils and the role of cyclooxygenases and lipooxygenases is not well defined. Further research is required to determine if one or more has a role to play in potentiation of a protective response.

\section{Conclusion}

This review summarizes the current state of knowledge of blocking the transmission of the malaria parasite from the vertebrate host to that of the mosquito. Understanding how apoptosis may be triggered in order to prevent fertilization of Plasmodium gametes inside the mosquito's midgut as well as what favours senescence instead of apoptosis within the human host will provide important information to facilitate the design of both effective therapies for infected patients and prophylactic transmission-blocking vaccines to restrict spread of malaria within a local community.

\section{Competing Interests}

The authors have declared no competing interests.

\section{Authors' Contributions}

SS drafted the manuscript, and AWT-R commented on and revised the paper. Both authors have reviewed and approved the final manuscript.

\section{References}

[1] US Census Bureau (2016) International Programs-World Population Clock. https://www.census.gov/population/international/

[2] World Health Organization (2015) World Malaria Report 2015. WHO, Geneva. http://www.who.int/malaria/publications/world-malaria-report-2015/en/

[3] Centers for Disease Control and Prevention (2016) Impact of Malaria. http://www.cdc.gov/malaria/malaria_worldwide/impact.html

[4] Johns Hopkins Malaria Research Institute (2016) About Malaria. http://malaria.jhsph.edu/about-malaria/

[5] Hay, S.I., Guerra, C., Tatem, A., Noor, A. and Snow, R. (2004) The Global Distribution and Population at Risk of Malaria: Past, Present, and Future. Lancet Infectious Diseases, 4, 327-336. http://dx.doi.org/10.1016/S1473-3099(04)01043-6

[6] Gallup, J.L. and Sachs, J.D. (2001) The Economic Burden of Malaria. American Journal of Tropical Medicine and Hygiene, 64, 85-96.

[7] Taylor-Robinson, A.W. (2014) Advancement towards an Approved Vaccine to Target Plasmodium falciparum Malaria. International Journal of Immunology, 2, 31-39. http://dx.doi.org/10.11648/j.iji.20140205.11

[8] Sherman, I.W. (1998) Malaria-Parasite Biology, Pathogenesis and Protection. ASM Press, Washington DC.

[9] Barik, T.K. (2015) Antimalarial Drug: From Its Development to Deface. Current Drug Discovery Technologies, 12, 225-228. http://dx.doi.org/10.2174/1570163812666150907100019

[10] Bradbury, R.S., Robertson, G., Norton, R.E. and Taylor-Robinson, A.W. (2014) Missing Malaria? Potential Obstacles 
to Diagnosis and Hypnozoite Eradication. Medical Journal of Australia, 201, 630-631. http://dx.doi.org/10.5694/mja14.00313

[11] Taylor-Robinson, A.W. (200) Species-Transcending Regulation of Malaria Parasitaemia. Parasitology Today, 16, 460461. http://dx.doi.org/10.1016/S0169-4758(00)01780-4

[12] de Roode, J.C., Pansini, R., Cheesman, S.J., Helinski, M.E., Huijben, S., Wargo, A.R., Bell, A.S., Chan, B.H., Walliker, D. and Read, A.F.(2005) Virulence and Competitive Ability in Genetically Diverse Malaria Infections. Proceedings of the National Academy of Sciences of the USA, 102, 7624-7628. http://dx.doi.org/10.1073/pnas.0500078102

[13] Josling, G.A. and Llinás, M. (2015) Sexual Development in Plasmodium Parasites: Knowing When It’s Time to Commit. Nature Reviews Microbiology, 13, 573-587. http://dx.doi.org/10.1038/nrmicro3519

[14] Gueirard, P., Tavares, J., Thiberge, S., Bernex, F., Ishino, T., Milon, G., Franke-Fayard, B., Janse, C.J., Ménard, R. and Amino, R. (2010) Development of the Malaria Parasite in the Skin of the Mammalian Host. Proceedings of the National Academy of Sciences of the USA, 107, 18640-18645. http://dx.doi.org/10.1073/pnas.1009346107

[15] Epstein, J.E., Rao, S., Williams, F., Freilich, D., Luke, T., Sedegah, M., de la Vega, P., Sacci, J., Richie, T.L. and Hoffman, S.L. (2007) Safety and Clinical Outcome of Experimental Challenge of Human Volunteers with Plasmodium falciparum-Infected Mosquitoes: An Update. Journal of Infectious Diseases, 196, 145-154. http://dx.doi.org/10.1086/518510

[16] Amino, R., Thiberge, S., Martin, B., Celli, S., Shorte, S., Frischknecht, F. and Ménard, R. (2006) Quantitative Imaging of Plasmodium Transmission from Mosquito to Mammal. Nature Medicine, 12, 220-224. http://dx.doi.org/10.1038/nm1350

[17] Tavares, J., Formaglio, P., Thiberge, S., Mordelet, E., Van Rooijen, N., Medvinsky, A., Ménard, R. and Amino, R. (2013) Role of Host Cell Traversal by the Malaria Sporozoite during Liver Infection. The Journal of Experimental Medicine, 210, 905-915. http://dx.doi.org/10.1084/jem.20121130

[18] Taylor-Robinson, A.W. (2002) Exoerythrocytic Malaria Vaccine Development: Understanding Host-Parasite Immunobiology Underscores Strategic Success. Expert Review of Vaccines, 1, 317-340. http://dx.doi.org/10.1586/14760584.1.3.317

[19] Taylor-Robinson, A.W. (2010) Regulation of Immunity to Plasmodium: Implications from Mouse Models for Blood Stage Malaria Vaccine Design. Experimental Parasitology, 126, 406-414. http://dx.doi.org/10.1016/j.exppara.2010.01.028

[20] Peymanfar, Y. and Taylor-Robinson, A.W. (2016) Plasmodium Sexual Stage Parasites Present Distinct Targets for Malaria Transmission-Blocking Vaccine Design. International Journal of Vaccines and Immunization, 2, 51-56.

[21] Carter, R. and Graves, P.M. (1988) Gametocytes. In: Wernsdorfer, W. and McGregor, I., Eds., Malaria: Principles and Practice of Malariology, Churchill Livingstone, London, 253-306.

[22] Carter, R. and Chen, D.H. (1976) Malaria Transmission Blocked by Immunization with Gametes of the Malaria Parasite. Nature, 263, 57-60. http://dx.doi.org/10.1038/263057a0

[23] Carter, R., Gwadz, R.W. and Green, I. (1979) Plasmodium gallinaceum: Transmission-Blocking Immunity in Chickens. I. The Effect of Anti-Gamete Antibodies in Vitro and in Vivo and Their Elaboration during Infection. Experimental Parasitology, 49, 196-208.

[24] Carter, R., Gwadz, R.W. and Green, I. (1981) Naturally Acquired Immunity and Antimalarial Antibodies in Relation to Infectivity to Mosquitoes in Endemic Plasmodium falciparum Malaria. Transactions of the 3rd Meeting of the Scientific Working Group on the Immunology of Malaria, Panama, June 1979, 105-121.

[25] Carlton, J.M., Angiuoli, S.V., Suh, B.B., Kooij, T.W., Pertea, M., Silva, J.C., et al. (2002) Genome Sequence and Comparative Analysis of the Model Rodent Malaria Parasite Plasmodium yoelii yoelii. Nature, 419, 512-519. http://dx.doi.org/10.1038/nature01099

[26] Pradel, G. (2007) Proteins of the Malaria Parasite Sexual Stages: Expression, Function and Potential for Transmission Blocking Strategies. Parasitology, 134, 1911-1929. http://dx.doi.org/10.1017/S0031182007003381

[27] Bhattacharyya, M.K. and Kumar, N. (2001) Effect of Xanthurenic Acid on Infectivity of Plasmodium falciparum to Anopheles stephensi. International Journal of Parasitology, 31, 1129-1133. http://dx.doi.org/10.1016/S0020-7519(01)00222-3

[28] Lakshmanan, V., Fishbaugher, M.E., Morrison, B., Baldwin, M., Macarulay, M., Vaughan, A.M., Mikolajczak, S.A and Kappe, S.H. (2015) Cyclic GMP Balance Is Critical for Malaria Parasite Transmission from the Mosquito to the Mammalian Host. mBio, 6, e02330-14. http://dx.doi.org/10.1128/mbio.02330-14

[29] Baker, D.A. and Kelly, J.M. (2004) Purine Nucleotide Cyclases in the Malaria Parasite. Trends in Parasitology, 20, 227-232. http://dx.doi.org/10.1016/j.pt.2004.02.007

[30] Gonçalves, D. and Hunziker, P. (2016) Transmission-Blocking Strategies: The Roadmap from Laboratory Bench to the 
Community. Malaria Journal, 15, 95. http://dx.doi.org/10.1186/s12936-016-1163-3

[31] Rener, J., Graves, P.M., Carter, R., Williams, J. and Burkot, T.R. (1983) Target Antigens of Transmission Blocking Immunity on Gametes of Plasmodium falciparum. The Journal of Experimental Medicine, 158, 976-981. http://dx.doi.org/10.1084/jem.158.3.976

[32] Vermeulen, A.N., Ponnudurai, T., Beckers, P.J.A., Verhave J.P., Smits, M.A. and Meuwissen, J.H.E.T. (1985) Sequential Expression of Antigens on Sexual Stages of Plasmodium falciparum Accessible to Transmission-Blocking Antibodies in the Mosquito. Journal of Experimental Medicine, 162, 1460-1476. http://dx.doi.org/10.1084/jem.162.5.1460

[33] Nikolaeva, D., Draper, S.J. and Biswas, S. (2015) Toward the Development of Effective Transmission-Blocking Vaccines for Malaria. Expert Review of Vaccines, 14, 653-680. http://dx.doi.org/10.1586/14760584.2015.993383

[34] Wu, Y., Sinden, R.E., Churcher, T.S., Tsuboi, T. and Yusibov, V. (2015) Development of Malaria Transmission-Blocking Vaccines: From Concept to Product. Advances in Parasitology, 89, 109-152. http://dx.doi.org/10.1016/bs.apar.2015.04.001

[35] Hurd, H., Carter, V. and Nacer, A. (2005) Interactions between Malaria and Mosquitoes: The Role of Apoptosis in Parasite Establishment and Vector Response to Infection. Current Topics in Microbiology and Immunology, 289, 185217. http://dx.doi.org/10.1007/3-540-27320-4_9

[36] Kerr, J.F., Wyllie, A.H. and Currie, A.R. (1972) Apoptosis: A Basic Biological Phenomenon with Wide Ranging Implications in Tissue Kinetics. British Journal of Cancer, 26, 239-257. http://dx.doi.org/10.1038/bjc.1972.33

[37] Gordeeva, A.V., Labas, Y.A. and Zvyagilskaya, R.A. (2004) Apoptosis in Unicellular Organisms: Mechanisms and Evolution. Biochemistry (Moscow), 69, 1055-1066. http://dx.doi.org/10.1023/b:biry.0000046879.54211.ab

[38] Paul, R.E., Brey, P.T. and Robert, V. (2002) Plasmodium Sex Determination and Transmission to Mosquitoes. Trends in Parasitology, 18, 32-38. http://dx.doi.org/10.1016/S1471-4922(01)02122-5

[39] Al-Olayan, E.B., Williams, G.T. and Hurd, H. (2002) Apoptosis in the Malaria Protozoan Plasmodium berghei: A Possible Mechanism for Limiting Intensity of Infection in the Mosquito. International Journal of Parasitology, 32, 1133-1143. http://dx.doi.org/10.1016/S0020-7519(02)00087-5

[40] Talman, A.M., Domarle, O., McKenzie, F.E., Ariey, F. and Robert, V. (2004) Gametocytogenesis: The Puberty of Plasmodium falciparum. Malaria Journal, 3, 24. http://dx.doi.org/10.1186/1475-2875-3-24

[41] Silvestrini, F., Alano, P. and Williams, J.L. (2000) Commitment to the Production of Male and Female Gametocytes in the Human Malaria Parasite Plasmodium falciparum. Parasitology, 121, 465-471. http://dx.doi.org/10.1017/S0031182099006691

[42] Carter, L.M., Schneider, P. and Reece, S.E. (2014) Information Use and Plasticity in the Reproductive Decisions of Malaria Parasites. Malaria Journal, 13, 115. http://dx.doi.org/10.1186/1475-2875-13-115

[43] Morlais, I., Nsango, S.E., Toussile, W., Abate, L., Annan, Z., Tchioffo, M.T., Cohuet, A., Awono-Ambene, P.H., Fontenille, D., Rousset, F. and Berry, A. (2015) Plasmodium falciparum Mating Patterns and Mosquito Infectivity of Natural Isolates of Gametocytes. PLOS ONE, 10, e0123777. http://dx.doi.org/10.1371/journal.pone.0123777

[44] Reece, S.E., Drew, D.R. and Gardner, A. (2008) Sex Ratio Adjustment and Kin Discrimination in Malaria Parasites. Nature, 453, 609-614. http://dx.doi.org/10.1038/nature06954

[45] Pollitt, L.C., Mideo, N., Drew, D.R., Schneider, P., Colegrave, N. and Reece, S.E. (2011) Competition and the Evolution of Reproductive Restraint in Malaria Parasites. The American Naturalist, 177, 358-367. http://dx.doi.org/10.1086/658175

[46] Hurd, H., Grant, K.M. and Arambage, S.C. (2006) Apoptosis-Like Death as a Feature of Malaria Infection in Mosquitoes. Parasitology, 132, S33-S47. http://dx.doi.org/10.1017/S0031182006000849

[47] Arambage, S.C., Grant, K.M., Pardo, I., Ranford-Cartwright, L. and Hurd, H. (2009) Malaria Ookinetes Exhibit Multiple Markers for Apoptosis-Like Programmed Cell Death in Vitro. Parasites \& Vectors, 2, 32. http://dx.doi.org/10.1186/1756-3305-2-32

[48] Ramiro, R.S., Alpedrinha, J., Carter, L., Gardner, A. and Reece, S.E. (2011) Sex and Death: The Effects of Innate Immune Factors on the Sexual Reproduction of Malaria Parasites. PLoS Pathogens, 7, e1001309. http://dx.doi.org/10.1371/journal.ppat.1001309

[49] Ahmed, A.M. and Hurd, H. (2006) Immune Stimulation and Malaria Infection Impose Reproductive Costs in Anopheles gambiae via Follicular Apoptosis. Microbes and Infection, 8, 308-315. http://dx.doi.org/10.1016/j.micinf.2005.06.026

[50] Dantzler, K.W., Ravel, D.B., Brancucci, N.M. and Marti, M. (2015) Ensuring Transmission through Dynamic Host Environments: Host-Pathogen Interactions in Plasmodium Sexual Development. Current Opinion in Microbiology, 26, 17-23. http://dx.doi.org/10.1016/j.mib.2015.03.005 